\title{
Nephrologists-led intervention increases utilisation of peritoneal dialysis in Singapore
}

\section{Dear Editor,}

Over the past decade, the utilisation of peritoneal dialysis (PD) among end-stage kidney disease (ESKD) patients in Singapore has been persistently low $(<20 \%){ }^{1}$ Difficulty in accessing PD catheter insertion services is considered one of the main obstacles to the growth of the PD population. ${ }^{2}$

Traditionally, PD catheter insertion has predominantly been provided by surgeons. Over the last 2-3 decades, there has been a growing trend of nephrologists directly participating in PD catheter insertion. However, interventional nephrology (IN) does not have a well-defined role in peritoneal dialysis (PD) service provision. There are reports and studies on a single "module" of IN practice in the field of PD, such as technical aspects of PD catheter insertion. ${ }^{3-5}$ More importantly, studies on holistic and multifaceted impacts on ESKD patients are absent, namely, dialysis modality of choice, dialysis service quality improvement and clinical outcomes from the establishment of IN practice. At Khoo Teck Puat Hospital in Singapore, we started IN services for PD in 2015. Here, we report the experience and outcomes of IN practice in PD service from a single centre.

This study is a review of our new approach to PD catheter insertion services. We postulated that an IN-led, multidisciplinary collaborative model of care for ESKD patients could improve utilisation and increase PD uptake as a dialysis modality of choice. This approach was based on: interventional nephrologistled PD catheter insertion service by mini-laparotomy; coordination of care by synchronising renal coordinator, nephrologist and anaesthetist visits; and PD initiated by instillation oflow-volume peritoneal dialysate (1-1.2L) for the first week, followed by incremental volume adjustment for patients indicated for urgent PD initiation.

From January 2011 to June 2020, 201 and 103 PD catheters were inserted by interventional nephrologists and urologists, respectively. All insertions were performed under a mini-laparotomy approach. Both groups had comparable age, ethnicity, body mass index, percentage of diabetes, hypertension, and ischaemic heart disease, while more patients with amputations had PD catheters performed by urologists. Both groups had similar dialysis adequacy.
Twenty-nine percent of our patients were able to initiate urgent PD within 2 weeks of catheter insertion by interventional nephrologists. The total number of PD catheter insertions (by both interventional nephrologists and urologists) increased by $116 \%$ from 70 (2011-2014) to 151 (2015-2018). The prevalence of PD patients in our programme increased by $217 \%$ after the initiation of IN services (48 in 2014 versus 104 in 2019). Between 2015 and 2019, 25-29\% of incident ESKD patients chose PD as the dialysis modality at our centre, compared to $10-23 \%$ between 2011 and 2014 when IN was not in place (Fig. 1, $P=0.015$ ).

Our PD catheter-related complication rate was similar in comparison to the International Society of Peritoneal Dialysis guidelines. ${ }^{6}$ For nephrologist-led insertion, the catheter adjustment-free survival was $95 \%$ and $94 \%$ at 12 months and 60 months, respectively. The 12-month and 60-month survival rates were $92 \%$ and $87 \%$, respectively, for urologist-led insertion (Table 1, Log-rank $P=0.165$ ).

IN practice has been growing steadily over the past 2-3 decades. There are isolated reports of centres in Asia offering IN services, but they are usually limited to 1-2 modules of IN. In our centre, IN services in tunnelled dialysis catheter insertion began in 2010, expanded to offer endovascular arteriovenous fistula/ graft procedures in late 2013, and finally graduated to

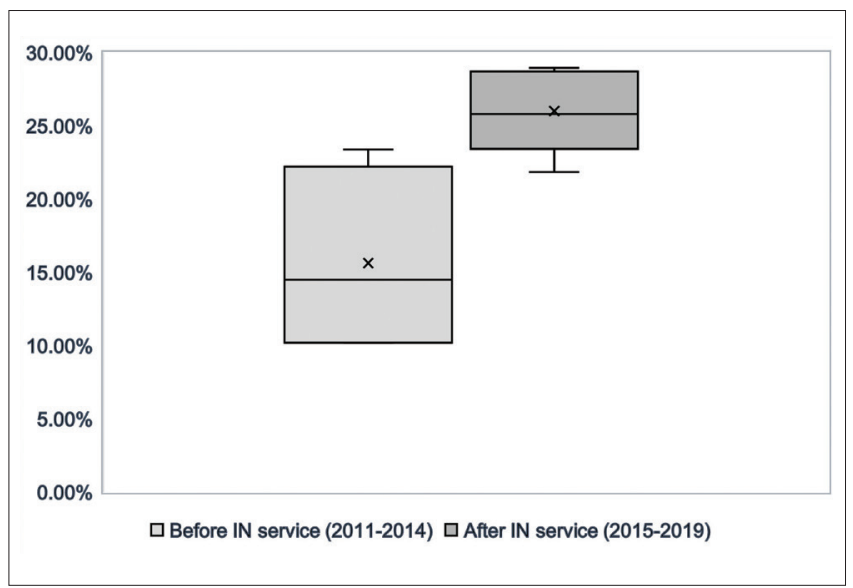

IN: interventional nephrology

Fig. 1. Comparison of the percentage of peritoneal dialysis uptake for incident end-stage kidney disease patients before and after the introduction of an interventional nephrology service $(P=0.015)$. 
Table 1. Peritoneal dialysis catheter-related complication rate from 2011 to 2019

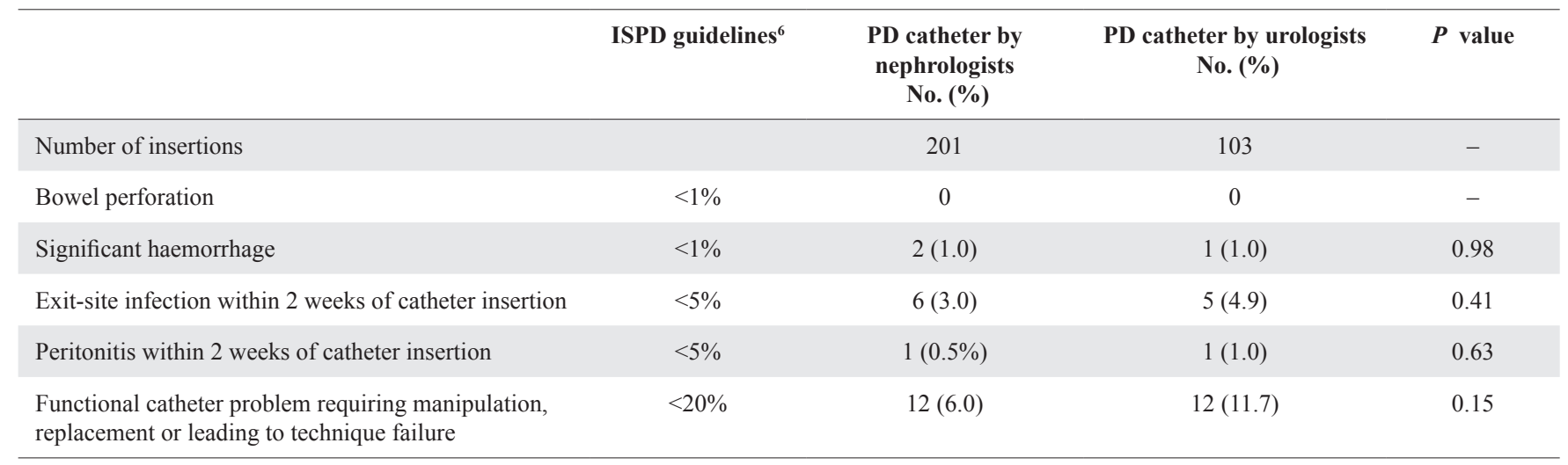

ISPD: International Society of Peritoneal Dialysis; PD: peritoneal dialysis

offer PD access services in 2015. By providing timely PD catheter insertion, effective coordination of care throughout the patient journey, and interim initiation of PD within 2 weeks of catheter insertion, the new collaborative model showed a sustained increase in incident PD uptake, with the resultant progressive increase in prevalent PD patients in our programme. There was also an increase in the percentage of incident PD patients by the modality of choice. Our short-term and long-term PD catheter survival rates were excellent, with a low complication rate.

We encountered challenges similar to those described by other authors who started IN services-accreditation, turf issues, lack of peer support and lack of facilities. Focusing on improving patient outcomes, engagement with other specialty colleagues, providing efficient services with reasonable safety and efficacy, teamwork, and support from the nephrology team are the keys to success for any IN programme.

PD catheter outcomes were excellent with a low rate of complications. We have respective standby support from urologists and general surgeons to address complications related to PD catheter insertion, including catheter readjustment and bowel perforation. Other complications, including exit-site infection and peritonitis, could be resolved by medical therapy. We also shared IN service experience of a rise in PD penetration once nephrologists insert PD catheters. ${ }^{3}$ Although multiple factors can influence PD utilisation, IN services genuinely provide a seamless care pathway for transition from predialysis counselling, education on dialysis modality of choice, assessment for PD eligibility, and insertion of PD catheters. This integrated care approach has also demonstrated success in multicentre studies. ${ }^{7}$ The role of IN in PD should not be confined to catheter insertion alone. Our service provision shows that coordination of care and collaboration with different disciplines are equally important as technical advancements in dialysis access management.

Urgent initiation of PD by the surgical placement of PD catheters is considered a reasonable alternative to the urgent start of haemodialysis for unplanned dialysis, provided that respective expertise and infrastructure are available. ${ }^{8}$ As demonstrated in our study, surgical placement of PD catheters is safe and effective for urgent PD initiation, potentiating PD utilisation as a dialysis modality of choice.

In conclusion, the role of IN in PD should be multifaceted and holistic. As demonstrated in our study, an integrated approach with collaborative and coordinated care orchestrated by interventional nephrologists provided steady and remarkable growth of PD patients in a dialysis programme. Our outcome of PD access confirms the safety and efficacy of advanced IN procedures when performed by appropriately trained nephrologists. We hope to test our collaborative approach in other institutional settings with different PD insertion techniques, interdisciplinary working cultures and dynamics to affirm our findings.

\section{REFERENCES}

1. Leo $\mathrm{CCH}$, Chan GC. Global perspectives in dialysis: Singapore. Kidney360 2020;1:1306-9.

2. Davies SJ. Peritoneal dialysis - current status and future challenges. Nat Rev Nephrol 2013;9:399-408. 
3. Goh BL, Ganeshadeva YM, Chew ES, et al. Does Peritoneal Dialysis Catheter Insertion by Interventional Nephrologists Enhance Peritoneal Dialysis Penetration? Sem Dial 2008;21:561-6.

4. Perl J, Pierratos A, Kandasamy G, et al. Peritoneal dialysis catheter implantation by nephrologists is associated with higher rates of peritoneal dialysis utilization: a population-based study. Nephrol Dial Transplant 2015;30:301-9.

5. Boujelbane L, Fu N, Chapla K, et al. Percutaneous versus surgical insertion of PD catheters in dialysis patients: a meta-analysis. J Vasc Access 2015;16:498-505.

6. Figueiredo A, Goh BL, Jenkins S, et al. Clinical practice guidelines for peritoneal access. Perit Dial Int 2010;30:424-9.

7. Ng EK, Goh BL, Chew SE, et al. Multicenter analysis on the impact of nephrologist-initiated catheter insertion program on peritoneal dialysis penetration. Semin Dial 2012;25:569-73.
8. Javaid MM, Khan BA, Subramanian S. Is surgical PD catheter insertion safe for urgent-start peritoneal dialysis? Semin Dial 2019;32:225-8.

Allen Yan Lun Liu,${ }^{1}{ }_{F R C P}$, Milind Nikam, ${ }^{2} M D$

${ }^{1}$ Khoo Teck Puat Hospital, Singapore

${ }^{2}$ Fresenius Medical Care, Singapore

Correspondence: Dr Allen Yan Lun Liu, Division of Renal Medicine, Department of General Medicine, Khoo Teck Puat Hospital, 90 Yishun Central, Singapore 768828 .

Email: liu.allen.yl@ktph.com.sg 